\title{
Novel application of an optical inspection system to determine the freshness of Scomber japonicus (mackerel) stored at a low temperature
}

\author{
Jeong-Wook Choi ${ }^{1} \cdot$ Myung-Kee Jang ${ }^{2} \cdot$ Chang-Wook Hong ${ }^{2} \cdot J^{u}-W o o n ~$ Lee $^{2} \cdot$ \\ Jae-Hyuk Choi ${ }^{2} \cdot$ Koth-Bong-Woo-Ri Kim ${ }^{3}$ - Xiaotong Xu${ }^{3} \cdot$ Dong-Hyun $\mathrm{Ahn}^{3}$. \\ Min-kyeong $\mathrm{Lee}^{4} \cdot$ Taek Jeong $\mathrm{Nam}^{1,4}$ (D)
}

Received: 21 August 2018/Revised: 10 May 2019/Accepted: 31 May 2019/Published online: 8 August 2019

(C) The Author(s) 2019

\begin{abstract}
This study evaluated the use of an optical inspection system (OIS) to determine the freshness of mackerel (Scomber japonicus). The correlations between the light reflection intensity (LRI) of mackerel eyes (determined using an OIS) and the volatile basic nitrogen content (VBN) and K-value were analyzed. After unloading at the harbor, the mackerel were stored at $4{ }^{\circ} \mathrm{C}$ for 9 days and the VBN, K-value, and LRI were determined at 3-day intervals. During storage, the LRI, VBN, and $\mathrm{K}$-value all increased. Furthermore, the LRI was correlated with the K-value and VBN. Therefore, although the LRI cannot be applied as an absolute standard for evaluating freshness, the LRI using an OIS is a suitable nondestructive method for evaluating freshness for quality and risk
\end{abstract}

\footnotetext{
Taek Jeong Nam

namtj@pknu.ac.kr

Jeong-Wook Choi

wook8309@naver.com

Myung-Kee Jang

leozzang@devicekorea.net

Chang-Wook Hong

cw0319@devicekorea.net

Ju-Woon Lee

sjwlee@naver.com

Jae-Hyuk Choi

cjh4954@naver.com

Koth-Bong-Woo-Ri Kim

kkbw10819@naver.com

Xiaotong $\mathrm{Xu}$

xiaotongmiki@gmail.com

Dong-Hyun Ahn

dhahn@pknu.ac
}

management in the processing industry when handling large numbers of fish.

Keywords Mackerel - Freshness - Nondestructive · Quality · Optical inspection system

\section{Introduction}

Freshness of fish is the most important aspect because it affects taste, texture and safety. Therefore, the priority value in purchasing fish is freshness. (Huang et al., 2011). However, consumers often cannot obtain objective information about the freshness, quality, and shelf life of mackerel during the sales process, and producers are

Min-kyeong Lee

3633234@hanmail.net

1 Institute of Fisheries Sciences, Pukyong National University, Busan 46041, Republic of Korea

2 Devicenet Research Institute, Anyang, Gyeonggi-do 14056, Republic of Korea

3 Department of Food Science and Technology/Institute of Food Science, Pukyong National University, Busan 48513, Republic of Korea

4 Department of Food Science and Nutrition, Pukyong National University, 45, Yongso-ro, Nam-Gu, Busan 48513, Republic of Korea 
unable to manage the quality and risk for all of the mackerel handled. Therefore, an improved freshness evaluation method is required to solve this problem and to increase consumer consumption of fish.

The quality and freshness of fish can be measured by using the levels of trimethylamine (TMA), volatile basic nitrogen (VBN), and thiobarbituric acid reactive substances (TBARS), and the K-value (Chang et al., 1998; Kuda et al., 2002). These measurement methods have been evaluated many times (Cheng et al., 2015). However, it takes considerable time to analyze the freshness and quality of fish during distribution, especially examining every fish because fish tissue is required for analysis.

Nondestructive inspection methods have occasionally been used to monitor and evaluate food quality (Daugaard et al., 2010; Huang et al., 2013; Paluchowski et al., 2016; Uddin et al., 2006), but there are no reports using optical inspection methods to measure the freshness of fish, especially the light reflection intensity (LRI) of eyes.

In this study, we used changes in the transparency of the eyes to measure freshness. Eye transparency and physicochemical analyses were conducted on mackerel stored at $4{ }^{\circ} \mathrm{C}$. Then, the correlations between the LRI and physicochemical results were evaluated to assess whether they were appropriate for measuring freshness.

\section{Materials and methods}

\section{Sample preparation}

Mackerel (Scomber japonicus) were purchased from a wholesale fishery market (Busan, Korea) just after being unloaded from a fishing boat. The mackerel samples were tagged with a numbered label on their tailfin and stored in a refrigerator at $4{ }^{\circ} \mathrm{C}$.

\section{Optical inspection}

\section{The optical inspection system (OIS)}

The OIS system consisted of an optical camera (Basler Ace A2000-50gc color CCD; Basler, Ahrensburg, Germany), lens (EDMUND 8.5 mm/F1.3, C-Mount; Edmund, Barrington, NJ, USA), and light source (white LED $20 \mathrm{~W}$ with a dome fixture; LFINE, Incheon, Korea). Images of the eyes were captured at $50 \mathrm{frames} / \mathrm{s}$ by a Gigabit Ethernet with Lab-view I-MAQ-dx Library (VDM; National Instruments, Austin, TX, USA), and the red-green-blue color (RGB) format intensity of each eye was processed in a 64-bit industrial PC environment.
Measurement of the eye reflection intensity

The LRI was measured with the OIS. The mackerel were put on a conveyer and passed through the optical system (Fig. 1A). As each mackerel moved under the optical camera and 11,700 lx light source, its whole body was captured by the optical camera $(2000 \mu$ s exposure $)$ and in order to uniformly illuminate the mackerel, the light reflector was used to induce dispersion of light. A program automatically selected the eye region (Fig. 1B) in mackerel picture using optical camera. Then, the RGB value of the eye image was measured according to the RGB color classification. In the analysis, only the red value was used because it had the closest relationship to the changes in freshness. The analyzed values were saved as the LRI after calculating the average and deviation.

\section{Chemical analysis}

\section{$V B N$}

The VBN content was determined by the Conway method following the guidelines of the Ministry of Food and Drug Safety (MFDS, 2016). Briefly, a $10 \mathrm{~g}$ mackerel sample and $50 \mathrm{~mL}$ of deionized distilled water (DDW) were put in a glass beaker and homogenized with a homogenizer (AM-7; Nihon Seiki Kaisha, Tokyo, Japan). The homogenate was stirred gently in a shaker for $10 \mathrm{~min}$. After shaking, the homogenate was allowed to stabilize for $5 \mathrm{~min}$ at room temperature (RT). This process was repeated three times. The homogenate was centrifuged at $2000 \times g$ for $10 \mathrm{~min}$ and the supernatant was filtered through Whatman No. 4 filter paper. The $\mathrm{pH}$ of the filtrate was adjusted to 4.0 by adding $5 \% \mathrm{H}_{2} \mathrm{SO}_{4}$ and the volume was made up to $100 \mathrm{~mL}$ with DDW. Then, $1.0 \mathrm{~mL}$ of $0.01 \mathrm{~N} \mathrm{H}_{2} \mathrm{SO}_{4}$ was put in the inner space of the Conway unit and $1.0 \mathrm{~mL}$ of saturated $\mathrm{K}_{2} \mathrm{CO}_{3}$ and the sample were added to the outer space of the Conway unit. The Conway unit was sealed immediately and incubated at $25{ }^{\circ} \mathrm{C}$, with $30 \%$ humidity, for $1 \mathrm{~h}$. After incubation, one drop of Brunswik reagent $(0.2 \mathrm{~g}$ of methyl red and $0.1 \mathrm{~g}$ of methylene blue in $300 \mathrm{~mL}$ of ethyl alcohol) was added to the inner space of the Conway unit. The solution was titrated using $0.01 \mathrm{~N} \mathrm{NaOH}$ and the VBN content was calculated using the following equation:

$\operatorname{VBN}(\mathrm{mg} / 100 \mathrm{~g})=0.14 \times[(A-B) \times F] / W \times 100 \times D$,

where $A$ is the volume $(\mathrm{mL})$ of $0.01 \mathrm{~N} \mathrm{NaOH}$ used for sample titration, $B$ is the volume $(\mathrm{mL})$ of $0.01 \mathrm{~N} \mathrm{NaOH}$ used for blank titration, $F$ is the factor of $0.01 \mathrm{~N} \mathrm{NaOH}$, $W$ is the sample weight (g), and $D$ is the dilution factor. 
(A)

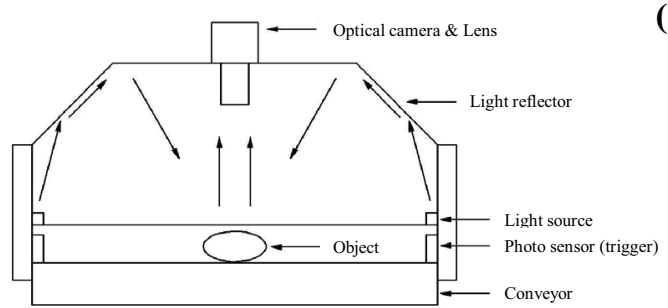

(B)

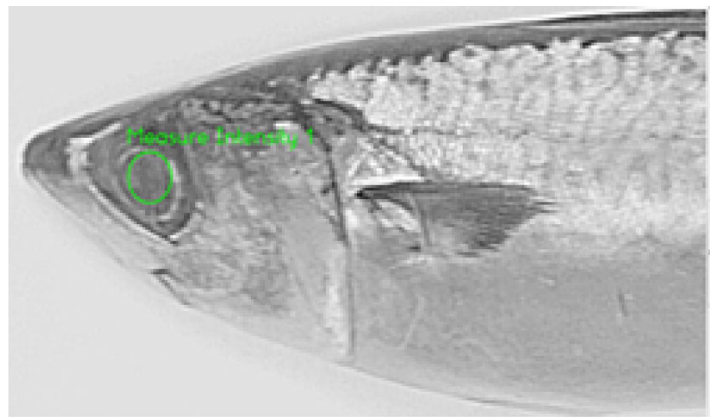

Fig. 1 (A) The optical inspection system (OIS) and (B) a photograph used for automatic eye recognition with the OIS (application of blue and green removal filter)

\section{K-value}

First, a $10.0 \mathrm{~g}$ mackerel sample was homogenated with $30 \mathrm{~mL}$ of chilled $10 \%$ perchloric acid in a glass beaker and allowed to stabilize for $30 \mathrm{~min}$ at RT. The homogenate was centrifuged at $2000 \times g$ for $20 \mathrm{~min}$ and the supernatant was collected. The precipitate was resuspended in chilled $10 \%$ perchloric acid and centrifuged under the same conditions. The supernatants from two extractions were pooled and filtered with Whatman No. 4 filter paper. The filtrate was neutralized with chilled $5 \mathrm{M} \mathrm{KOH}$ to $\mathrm{pH} 6.5$ and the final volume adjusted to $100 \mathrm{~mL}$ using DDW. Then, the resulting solution was filtered through a $0.45 \mu \mathrm{m}$ membrane (Cat. no. 4408; PALL Corp., Ann Arbor, MI, USA), and $10.0 \mu \mathrm{L}$ of the filtrate were injected into a high-performance liquid chromatography (HPLC) instrument (1100 Series; Agilent Technologies, Palo Alto, CA, USA) to analyze nucleic acid-related compounds [NARCs; adenosine triphosphate (ATP), adenosine diphosphate (ADP), adenosine monophosphate (AMP), inosine monophosphate (IMP), inosine, (HxR), and hypoxanthine (Hx)]. The chemical reagents used as NARC standards were purchased from Sigma Aldrich (St. Louis, MO, USA).

The analytical conditions for HPLC were as follows: ultraviolet (UV) detection at $254 \mathrm{~nm}$, the range of absorbed dose (absorbance units full scale; AUF) was 0.5 , $\mu$ Bondapak $\mathrm{C}_{18}$ column $(3.9 \mathrm{~mm}$ i.d. $\times 300 \mathrm{~mm}$; Waters, Milford, MA, USA), column chamber temperature $40{ }^{\circ} \mathrm{C}$, flow rate $2.0 \mathrm{~mL} / \mathrm{min}$, and mobile phase $1 \%$ tri-ethylamine $(\mathrm{pH} 6.5)$ adjusted with $10 \% \quad \mathrm{H}_{3} \mathrm{PO}_{4}$. The $\mathrm{K}$-value was calculated using the following equation:

$$
\mathrm{K}(\%)=\frac{(\mathrm{HxR}+\mathrm{Hx})}{\begin{array}{l}
\mathrm{ATP}+\mathrm{ADP}+\mathrm{AMP}+\mathrm{IMP}+\mathrm{HxR}+\mathrm{Hx} \\
\times 100 .
\end{array}}
$$

\section{Statistical analysis}

Values are presented as mean \pm standard deviation (SD). The data were analyzed with SPSS ver. 18.0 (SPSS Inc.,
Chicago, IL, USA) using a one-way analysis of variance followed by Duncan's multiple range test. Furthermore, Pearson correlation analysis was used to assess the correlation of freshness between the LRI and chemical analysis results (VBN, K-value). $P$-values $<0.05$ were considered to indicate statistical significance.

\section{Results and discussion}

\section{Evaluation of freshness with chemical analyses}

When the mackerel were stored at $4{ }^{\circ} \mathrm{C}$, the VBN content increased rapidly during storage from $6.11 \mathrm{mg} / 100 \mathrm{~g}$ at day 0 to $21.26 \mathrm{mg} / 100 \mathrm{~g}$ after 9 days (Fig. 2A). Generally, VBN content is $5-10 \mathrm{mg} / 100 \mathrm{~g}$ when the fresh fish, in the moderately fresh $15-25 \mathrm{mg} / 100 \mathrm{~g}$; when the fish started to spoil, it increased to $30-40 \mathrm{mg} / 100 \mathrm{~g}$, exceeding $50 \mathrm{mg} /$ $100 \mathrm{~g}$ when it was spoiled (Tsai et al., 2018).

The $\mathrm{K}$-value is an important index of fish freshness based on nucleotide degradation. During autolysis in fish, ATP gradually breaks down into ADP, AMP, IMP, HxR, and $\mathrm{Hx}$ as a result of enzymatic and microbial activity (Lowe et al., 1993). As degradation progresses, the HxR and Hx contents increase markedly (Hamada-Sato et al., 2005). When determining freshness using the $\mathrm{K}$-value, fresh fish should have a value $<10 \%$, sashimi $10-20 \%$, moderately fresh $20-50 \%$, raw material for processing $35-60 \%$, and spoiled material $>60 \%$ (Huang et al., 2015). The K-value of mackerel stored at $4{ }^{\circ} \mathrm{C}$ increased rapidly during storage (Fig. 2B), from $4.21 \%$ initially to $51.71 \%$ (about 12.28 times) after 9 days.

\section{Changes in LRI}

During storage at $4{ }^{\circ} \mathrm{C}$, the LRI of mackerel increased with the length of storage (Fig. 2C), from 26.04 at day 0 to 37.5 (1.4 times), 40.62 (1.5 times), and 44.55 (1.7 times) at days 3,6 , and 9 , respectively. Initially, the mackerel eyes were 

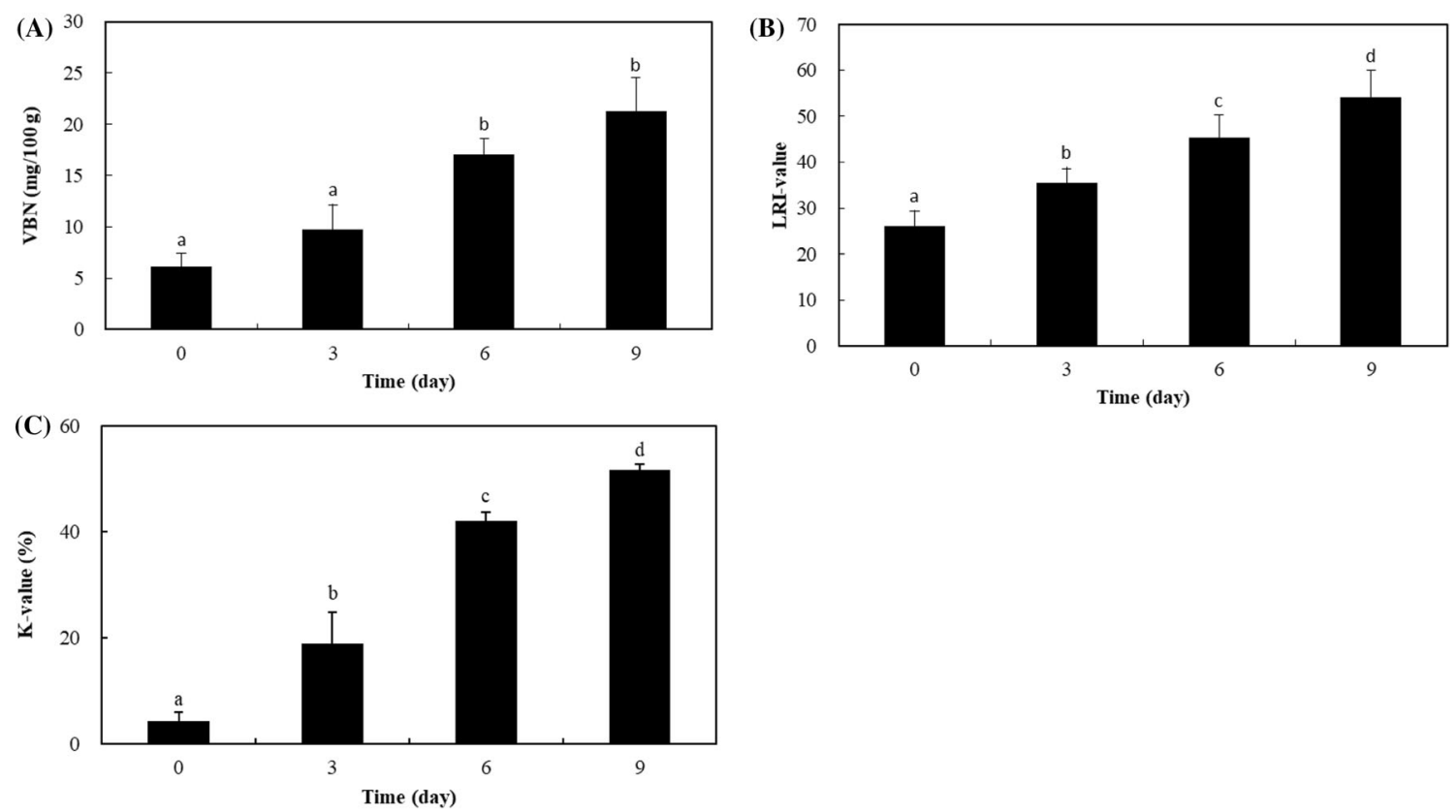

Fig. 2 (A) VBN content, (B) K-value, and (C) LRI of mackerel during storage at $4{ }^{\circ} \mathrm{C}$ for 9 days. The values are the mean \pm SD. Different lowercase letters indicate significant differences at $p<0.05$

Table 1 The Pearson correlations between the LRI and the VBN content and $\mathrm{K}$-value in mackerel during storage at $4{ }^{\circ} \mathrm{C}$ for 9 days

\begin{tabular}{lll}
\hline & VBN content & K-value \\
\hline LRI & $0.911^{\mathrm{a}}$ & $0.906^{\mathrm{b}}$ \\
\hline
\end{tabular}

Values are the mean \pm SD

Different lowercase letters indicate significant differences at $p<0.05$

transparent and the LRI was low. During storage, the LRI increased gradually. It is thought that the light-reflecting intensity of the fish eyes was inhibited by the change in turbidity of the eye lens. The LRI was highly correlated with the K-value $(r=0.906)$ and VBN content $(r=0.911)$ (Table 1). However, it was impossible to estimate the $\mathrm{K}$-value or VBN content in reverse based on the LRI only. Consequently, it is clear that the LRI is of limited value for evaluating freshness. Nevertheless, the LRI can be used to identify the freshness of fish to manage potential risks, and it has the advantage of being a nondestructive method that can potentially be applied when processing large numbers of mackerel.

Acknowledgements This research was part of a project titled "Technological development of an intelligent quality evaluation system for marine products (20150583)", funded by the Ministry of Oceans and Fisheries, Korea.

Open Access This article is distributed under the terms of the Creative Commons Attribution 4.0 International License (http://crea tivecommons.org/licenses/by/4.0/), which permits unrestricted use, distribution, and reproduction in any medium, provided you give appropriate credit to the original author(s) and the source, provide a link to the Creative Commons license, and indicate if changes were made.

\section{References}

Chang KLB, Chang J, Shiau CY, Pan BS. Biochemical, microbiological, and sensory changes of sea bass (Lateolabrax japonicus) under partial freezing and refrigerated storage. J. Agric. Food Chem. 46: 682-686 (1998)

Cheng JH, Sun DW, Pu H, Zhu Z. Development of hyperspectral imaging coupled with chemometric analysis to monitor $\mathrm{K}$ value for evaluation of chemical spoilage in fish fillets. Food Chem. 185: 245-253 (2015)

Daugaard SB, Adler-Nissen J, Carstensen JM. New vision technology for multidimensional quality monitoring of continuous frying of meat. Food Control 21: 626-632 (2010)

Hamada-Sato N, Usui K, Kobayashi T, Imada C, Watanabe E. Quality assurance of raw fish based on HACCP concept. Food Control 16: 301-307 (2005)

Huang H, Liu L, Ngadi MO, Gariépy C. Prediction of pork marbling scores using pattern analysis techniques. Food Control 31: 224-229 (2013)

Huang X, Lv R, Yao L, Guan C, Han F, Yeye E. Non-destructive evaluation of total volatile basic nitrogen (TVB-N) and K-values in fish using colorimetric sensor array. Anal. Methods 7: 1615-1621 (2015)

Huang X, Xin J, Zhao J. A novel technique for rapid evaluation of fish freshness using colorimetric sensor array. J. Food Eng. 105: 632-637 (2011)

Kuda T, Matsumoto C, Yano T. Changes in acid and alkaline phosphatase activities during the spoilage of raw muscle from horse mackerel Trachurus japonicus and gurnard Lepidotriga microptera. Food Chem. 76: 443-447 (2002)

Lowe T, Ryder J, Carragher J, Wells R. Flesh quality in snapper, Pagrus auratus, affected by capture stress. J. Food Sci. 58: 770-773 (1993) 
MFDS (Ministry of Food and Drug Safety). Test methods in the Food Code. Seoul, Korea. pp. 221-222 (2016)

Paluchowski LA, Misimi E, Grimsmo L, Randeberg LL. Towards automated sorting of Atlantic cod (Gadus morhua) roe, milt, and liver-Spectral characterization and classification using visible and near-infrared hyperspectral imaging. Food Control 62: 337-345 (2016)

Tsai CL, Shiau CY, Sung WC. Effects of blanching and refrigerated storage on quality attributes of hybrid abalone (Haliotidae discushannai X $H$. diversicolor diversicolor). J. Food Process. Pres. 42: e13608 (2018)

Uddin M, Okazaki E, Ahmad MU, Fukuda Y, Tanaka M. NIR spectroscopy: A non-destructive fast technique to verify heat treatment of fish-meat gel. Food Control 17: 660-664 (2006)

Publisher's Note Springer Nature remains neutral with regard to jurisdictional claims in published maps and institutional affiliations. 